\title{
Spektrum Ophthalmologie - wissenswert, kompakt, anregend
}

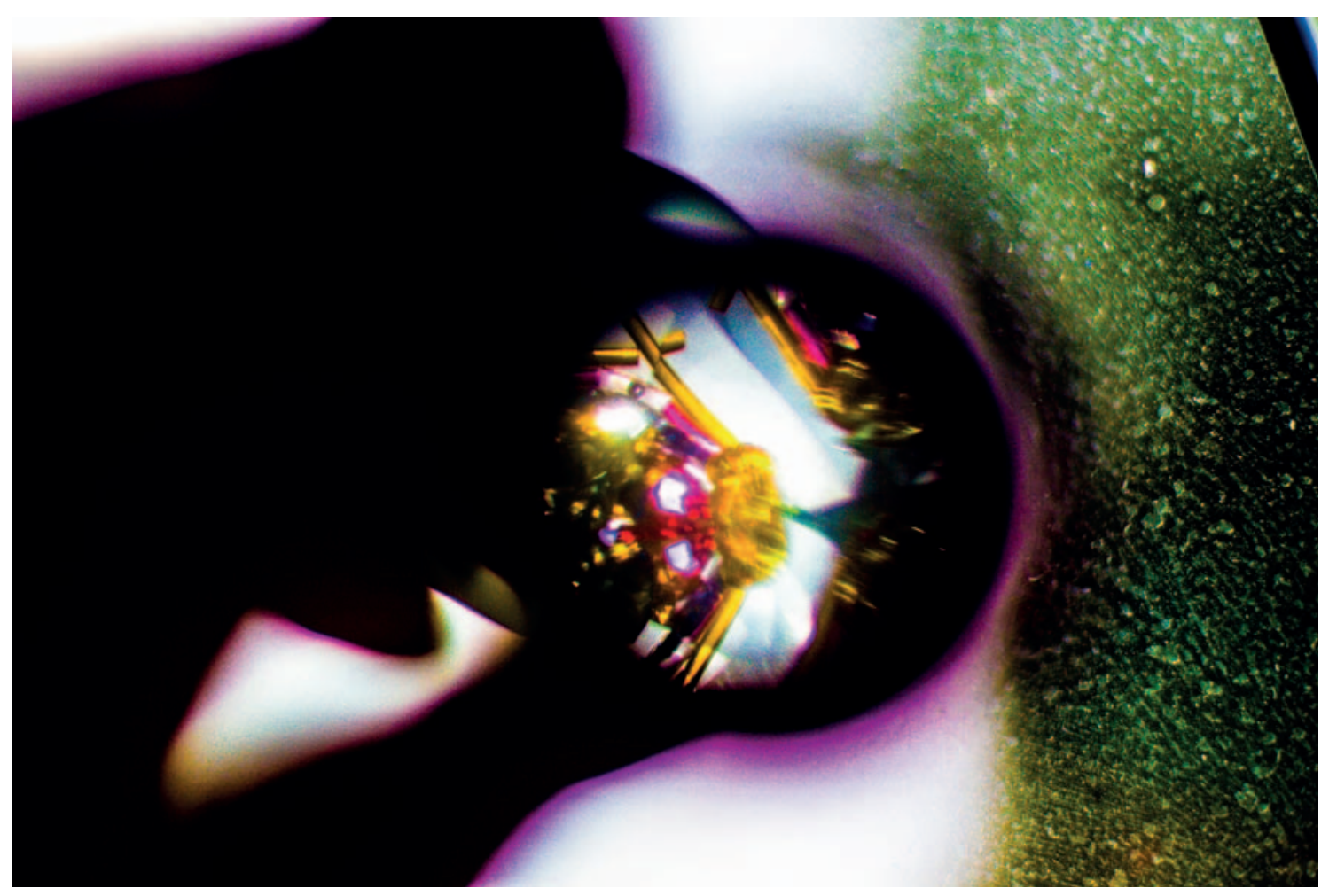

Woche des Sehens

\section{Seh(n)sucht}

«lch bin blind. Wie, weiß ich nicht. Mein Neffe hat mir zum Geburtstag ein Kaleidoskop geschenkt. Er sagte, ich solle damit ins Licht sehen. Er meinte, dass ich dann bestimmt etwas sehen kann. Ich nahm es und ... naja ich wusste nicht was Licht bedeutet.»

Die Fotografie von Vincent Rotunno wurde mit dem Sonderpreis der Woche des Sehens in der Kategorie «Bestes Bild eines blinden oder sehbehinderten Fotografen» ausgezeichnet. Gesucht wurden anspruchsvolle Fotografien, die die Themen Sehen, Blindheit und Sehbehinderung nicht nur bildlich, sondern auch kreativ und ansprechend darstellen. Mehr als spezielle Kenntnisse im Umgang mit Kamera und Objektiven waren hier der Ideenreichtum und die Fantasie gefragt. «ldeenreichtum und Fantasie ... was stellen wir uns vor wie ein Blinder sieht?», fragt der sehbehinderte Fotograf, «lch weiß es nicht, aber ich weiß wie Licht für uns in absoluter Nähe fühlbar wird - in diesem Bild. Sonnenstrahl, Kaleidoskop und Sehnerv bilden eine Linie.» Das
Bild wurde durch ein Kaleidoskop aufgenommen. Mit direkten Blick in die Sonne und durch die Gardine eines Schlafzimmers. Es zeigt die Ästhetik der Farben und der Dunkelheit. Sehnsucht und Sehsucht ganz nah beieinander. Nicht nur für Blinde, sondern auch für Sehende.

www.woche-des-sehens.de $\rightarrow$ Veranstaltungen $\rightarrow$ Fotowettbewerb

\section{KARGER}

Fax +497614520714 information@karger.com www.karger.com (c) 2017 S. Karger GmbH, Freiburg 
Deutsche Gesellschaft für Neurologie

Update Neuroophthalmologie: das Auge als Fenster zum Gehirn

«Mit modernen bildgebenden Verfahren lassen sich sowohl vaskuläre als auch neuronale Veränderungen der Retina, die Teil des Zentralen Nervensystems ist, darstellen und analysieren», sagt Professor Tjalf Ziemssen, Neurologe mit ophthalmologischen Wurzeln am Universitätsklinikum Dresden. «Viele neurologische Erkrankungen können wir damit heute am Auge ablesen.» Die in den letzten Jahren methodisch enorm verbesserte optische Kohärenztomographie (OCT) ist wesentlich aussagekräftiger als eine konventionelle Ophthalmoskopie: Man kann alle Retinaschichten in mikroskopischer Auflösung darstellen und in wenigen Sekunden abscannen (Abb. 1). Die gemessenen Veränderungen korrelieren erstaunlich gut mit degenerativen Hirnveränderungen bei chronischen neurologischen Erkrankungen: z.B. die Dicke der retinalen Faserschicht (RNFL) mit dem Hirnvolumen, die Dicke der inneren Körnerschicht (INL) mit dem Volumen aller in der Kernspintomographie (MRT) dargestellten T2-hyperintensen Entzündungsherde und die Dicke der äußeren Körnerschicht (ONL) mit der fortschreitenden Neurodegeneration bei Multipler Sklerose (MS). Diese Erkenntnisse konnten auch auf andere neurologische Erkrankungen übertragen werden, so gibt es Veränderungen der RNFL bei der Alzheimer-Demenz oder Veränderungen der ONL und der Dicke der Ganglionzellschicht bei Parkinson-Patienten.

\section{Struktur und Funktion parallel analysieren} Herausragende Bedeutung hat die Retinadiagnostik bereits jetzt bei MS. Mittels OCT können degenerative Veränderungen der Retina in der Folge von Optikusneuritiden, aber auch unabhängig hiervon nachge- wiesen werden. Mit rezenten Post-Prozessierungsverfahren können zudem nicht nur die retinale Nervenfaserschicht, sondern auch tiefergelegene neuronale Schichten der Netzhaut reliabel vermessen werden. Hierdurch können beispielsweise axonale von neuronalen Degenerationen (Atrophie) getrennt prozessiert und mit MRT-Parametern zur Atrophie (z.B. graue Substanz) korreliert werden. «Das visuelle System ist somit ideal, um den Prozess der Neurodegeneration, Neuroprotektion oder sogar Neuroregeneration zu evaluieren und darzustellen, zumal man Struktur und Funktion parallel erfassen und analysieren kann», fasst Ziemssen zusammen und ergänzt: «Die Möglichkeit, neuroregenerative Prozesse anhand der unterschiedlichen Untersuchungsverfahren des optischen Systems darstellen zu können, haben wir schon mehrfach in klinischen Studien zur Evaluation neuer Medikamente genutzt.» www.dgn.org $\rightarrow$ Pressemitteilungen 2016

Abb. 1. Das Bildresultat einer OTC-Angiografie liefert einen automatisierten segmentalen Algorhythmus. Elf unterschiedliche Schichten der Retina können auf diese Weise sichtbar gemacht und zur neurologischen Diagnose genutzt werden. OTC Angiography and Macular Diseases. Dev Ophthalmol. Basel, Karger, 2016, vol 56, pp 30-36.

\section{Universitätsmedizin Mainz}

\section{Schonende Behandlung des Glaukoms ohne Perforation der Bindehaut}

Ein neues minimal-invasives OP-Verfahren bei der Behandlung des Glaukoms (Abb. 1.) kommt in der Augenklinik und Poliklinik der Universitätsmedizin Mainz zum Einsatz, mit dem sich auf besonders schonen-

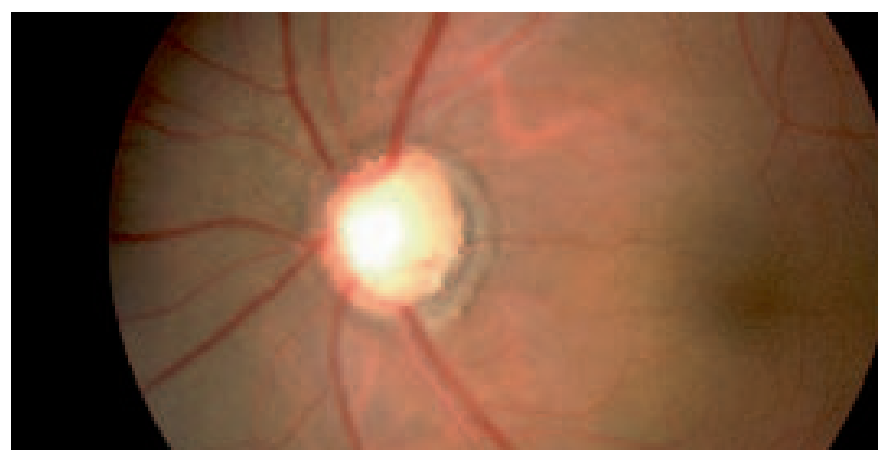

Abb. 1

Fortgeschrittene Glaukomerkrankung mit signifikanter Aushöhlung des Sehnervenkopfes. @ Universitätsmedizin Mainz. handelt sich um ein Gel-Implantat - über einen 1,8 mm langen Schnitt in das Trabekelwerk des Auges eingeführt. Dieses Implantat kommt unter der Bindehaut zum Liegen. Über diesen Schlauch, der ungefähr so dick wie ein Haar ist, sickert das Kammerwasser aus dem Auge unter die Bindehaut. Dieses Drainageprinzip trägt zur Regulierung des Augeninnendrucks bei.» Ähnlich wie bei einer konventionellen fistulierenden Glaukom-OP entsteht bei der neuen minimal-invasiven OP ein Sickerkissen. Über dieses Sickerkissen lässt sich Kammerwasser unter die Bindehaut drainieren. Die so eingeleitete Absenkung des Augeninnendrucks verhindert, dass die Schädigung des Sehnervs voranschreitet.

www.unimedizin-mainz.de $\rightarrow$ Pressemitteilungen 2016 
Abteilung für Wissenschaft und Technologie, Französische Botschaft in

Deutschland

\section{Optimierung von Netzhautprothesen}

Dank Netzhautprothesen können erblindete Menschen zwar Lichtsignale erkennen, aber kein klares Bild sehen. Um die Bildauflösungsgrenzen zu verstehen und das System zu optimieren, verglichen die Forscher des französischen Zentrums für wissenschaftliche Forschung (CNRS) die Ant-

\section{Carstens-Stiftung \\ Prüfung der Evidenz: \\ Komplementärmedizin in der Augenheilkunde}

Sind komplementäre und alternative Methoden wirksam in der Versorgung von $\mathrm{Pa}$ tienten mit Glaukom, Katarakt oder AMD? Wissenschaftler der Universität Witten/ Herdecke untersuchten dies im Rahmen eines systematischen Reviews [1] mit dem Ziel, erstmalig das gesamte Spektrum komplementärmedizinischer Verfahren in der augenärztlichen Anwendung zu erfassen. Sie schlossen Verfahren wie die Phytotherapie, Akupunktur/Akupressur, Biofeedback

\section{Ärztliches Zentrum für Qualität in der Medizin \\ Patientenleitlinie zur Prävention und Therapie von Netzhautkom- plikationen bei Diabetes}

Nach der Neuauflage der Nationalen Versorgungsleitlinie «Prävention und Therapie von Netzhautkomplikationen bei Diabetes» ist jetzt auch die laienverständliche Patientenleitlinie durch das Ärztliche Zentrum für Qualität in der Medizin ( öffentlicht worden. Sie übersetzt die Empfehlungen der Experten in eine allgemeinverständliche Sprache. Außerdem, so das ÄZQ weiter, bietet sie Menschen mit Diabetes und deren Angehörigen wichtige Informationen zum Umgang mit der Krankheit und Unterstützung beim Gespräch mit dem Arzt.

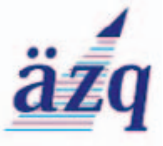

www.patienten-information.de $\rightarrow$ Kurzinformationen $\rightarrow$ Diabetes wort des visuellen Systems im Hirn eines Nagetiers auf natürliche visuelle Reize und auf durch die Prothese hervorgerufenen Reize. Diese Studie zeigte, dass die Prothese die Sehrinde des Nagetiers zwar an der richtigen Stelle aktiviert, die Aktivierungen jedoch viel zu groß und zu langgestreckt sind. Grund dafür ist eine übermäßige elektrische Verteilung in der Elektrodenmatrix, die ins Auge implantiert wurde und mit der Netzhaut verankert ist, sowie die unerwünschte Aktivierung von Sehfasern in der

sowie andere alternative Heilverfahren in ihre Untersuchung ein. Studien zu Nahrungsergänzungsmitteln (Vitamine oder Antioxidantien) wurden nicht berücksichtigt, da hierzu bereits eine gute Informationsbasis existiert. Zwar sind die verschiedenen Methoden nur eingeschränkt miteinander vergleichbar. Dennoch kann man auf der Suche nach evidenzbasierten, komplementärmedizinischen Methoden hier einen Eindruck erhalten, welche Behandlungsmethode sich bei welcher der drei untersuchten Augenerkrankungen als aussichtsreich erweisen könnte.

Max-Planck-Instituts für molekulare

Zellbiologie und Genetik

\section{Das Sehen verstehen}

Der Zebrafisch dient Forschern als Modell für die humane Netzhaut: Während der Embryonalentwicklung wandern die retinalen Ganglienzellen durch die entstehende Netzhaut und bilden dann ihre erste Schicht. Später entsteht aus diesen Zellen der Sehnerv.

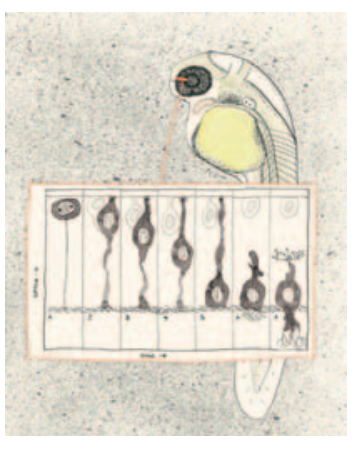

(c) Julia Eichhorn, Max-Planck-Institut für Molekulare Zellbiologie und Genetik

www.mpi-cbg.de $\rightarrow$ Aktuelles \& Veranstaltungen $\rightarrow$
Nähe der Zielzellen. Aus diesen Ergebnissen konnten die Forscher die Eigenschaften der Prothese/Netzhaut-Schnittstelle ableiten und so die Leistung der Prothese verbessern.

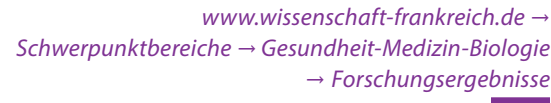

\section{Literatur}

1 Welte AK, Hahn U, Büssing A, Krummenauer F: Ergebnisse eines systematischen Reviews zu Einsatz und berichtetem therapeutischen Nutzen komplementärmedizinischer Methoden in der Augenheilkunde. Klin Monbl Augenheilkd;DOI:10.1055/s-0042-106901.

\section{aturheilkunde und Homôopathie \\ Erforschen. Erklären. Erleben.}

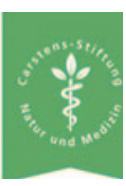

www.carstens-stiftung.de $\rightarrow$ Nachrichtenagentur $\rightarrow$ Studien kurz und knapp

\section{American Academy of Ophtalmology} Wikipedia für die Augen

EyeWiki ist eine englischsprachige AugenEnzyklopädie. In dem moderierten Forum findet der Leser Publikationen und Videovon Augenärzten zum großen Spektrum der Augenerkrankungen, ihrer Diagnostik und Therapie. Zudem ist jeder qualifizierte Augenarzt aufgerufen, hier selbst Beiträge zu verfassen oder andere Beiträge zu kommentieren. EyeWiki wird von einer Kollaboration der American Academy of Ophthalmology mit mehr als einem Dutzend Fachgesellschaften betrieben.

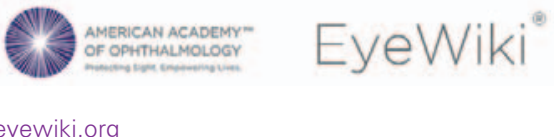

Die Inhalte dieser Rubrik sind von der Redaktion ausgewählt und bearbeitet. Die Auswahl erfolgt unabhängig von der Industrie und in Abstimmung mit dem Herausgeber der Zeitschrift. 